\section{Innovative antidepressants arrive}

\section{The FDA approved Johnson \& Johnson's} esketamine for treatment-resistant depression.

Esketamine, the first antidepressant in a new class to hit the market in decades, is an enantiomer of the analgesic and 'party drug' ketamine. It offers a fast-acting option to patients resistant to other antidepressants.

The nasal spray drug was approved on the basis of mixed clinical trial results. In one shortterm 4-week study, it showed a statistically significant effect compared with placebo on the severity of depression, with some effects seen within 2 days of treatment. Two other short-term trials did not meet their primary efficacy aims. In a longer-term maintenance-of-effect trial, patients in stable remission had a statistically significantly longer time to relapse of depressive symptoms than patients in a control arm.

An independent advisory panel voted 14 to 2 in favour of approval, with 1 abstention. Panelists noted concerns about the mixed results and the effect size, but were also swayed by the novelty of the drug and the unmet need in this setting. The drug carries a black box warning, noting the risks of sedation, dissociation, abuse and misuse. The drug must consequently be administered in clinics.

Researchers are still working to understand how esketamine improves moods. Whereas most other antidepressants modulate signalling by acting on the monoamine neurotransmitters serotonin, dopamine and noradrenaline, esketamine's effect was initially linked to its activity as an NMDA receptor antagonist. That picture has since become blurred, however. A slew of would-be competitor NMDA-modulating candidates, from companies including Pfizer, AstraZeneca and Merck \& Co., have failed in the clinic and have been discontinued.

One leading hypothesis is that esketamine might also act through a glutamate receptor called the AMPA receptor, which can stimulate neuronal rewiring.

In March, Allergan announced that its rapastinel, a partial NMDA agonist that also activates AMPA receptors, failed in three phase III trials in major depressive disorder (MDD).

The company said it will make a decision about whether to pursue further development of the drug in MDD and suicide later this year.

Separately, the FDA approved Sage Therapeutics' brexanolone as the first treatment for post-partum depression (PPD).

Brexanolone is an allosteric $\mathrm{GABA}_{\mathrm{A}}$ receptor modulator, and is administered via continuous intravenous infusion over 60 hours. Its approval was based on two placebo-controlled trials in patients with severe and moderate PPD, in which it was superior to placebo at improving depressive symptoms after a first infusion. The drug also carries a risk of serious harm owing to the sudden loss of consciousness, and it must be administered in a clinic so that patients can be monitored.

Sage's oral follow-on drug SAGE-217 is in phase III trials for MDD and PPD, and in phase II trials in other indications.

Asher Mullard

\section{Anti-amyloid failures stack up as Alzheimer antibody flops}

Partners Biogen and Eisai have terminated two phase III trials of their anti-amyloid antibody aducanumab in Alzheimer disease.

Despite repeated failures with other anti-amyloid antibodies, amyloid advocates had high hopes for aducanumab because of preliminary clinical results. An interim analysis of a phase lb trial in patients with mild or prodromal disease showed that treatment reduced brain $A \beta$ levels in a dose- and timedependent manner, investigators reported in 2016. This effect was accompanied by an apparent slowing of cognitive decline. The antibody's purported effect was attributed in part to its ability to selectively bind $A \beta$ aggregates such as soluble oligomers and insoluble fibrils, rather than monomers.
These hopes came crashing down in March when aducanumab's data monitoring committee found in a futility analysis that the phase III trials of the antibody were "unlikely to meet their primary end point".

The partners are still assessing the data and considering their options for a planned phase III trial in a secondary prevention setting.

This failure marks yet another setback for much beleaguered amyloid-modulating therapies (TABLE 1). At least four anti-amyloid antibodies have failed in phase III trials in different Alzheimer disease settings. Three BACE inhibitors and two $\gamma$-secretase inhibitors, which act on amyloid processing, have also bombed out in would-be pivotal trials. In some cases, these treatments were associated with worsening cognition. Many more related candidates have failed in phase II trials.

Some drug hunters still have hope that anti-amyloids will deliver, either in earlier disease settings or with better-optimized properties. The day after Eisai and Biogen announced the aducanumab failure, they advanced BAN2401 into a phase III trial in early disease. This antibody selectively binds to $A \beta$ protofibrils.

Others are increasingly calling for the exploration of alternative treatment strategies. These include tau-modulating drugs, microglia-targeted candidates and infectious disease-related agents.

Asher Mullard

Table 1 | Select list of failed phase III trials based on the amyloid hypothesis of Alzheimer disease

\begin{tabular}{lllll}
\hline Drug name & Sponsor & Properties & Alzheimer disease settings & Reasons for discontinuation \\
\hline Aducanumab & Biogen/Eisai & Anti-A $\beta \mathrm{mAb}$ & Early & Lack of efficacy \\
\hline Solanezumab & Eli Lilly & Anti-A $\beta \mathrm{mAb}$ & Mild to moderate, mild and prodromal & Lack of efficacy and strategic \\
\hline Bapineuzumab & Elan/Wyeth/Pfizer & Anti-A $\beta \mathrm{mAb}$ & Mild to moderate & Lack of efficacy \\
\hline Immunoglobulin & Baxter & Anti-A $\beta \mathrm{mAb}$ & Mild to moderate & Lack of efficacy \\
\hline Lanabecestat & AstraZeneca/Eli Lilly & BACE inhibitor & Early and mild & Lack of efficacy \\
\hline Atabecestat & Janssen & BACE inhibitor & Asymptomatic at risk & Toxicity \\
\hline Verubecestat & Merck \& Co. & BACE inhibitor & Mild to moderate and prodromal & Lack of efficacy \\
\hline Semagacestat & Eli Lilly & $\gamma$-secretase inhibitor & Mild to moderate & Toxicity and lack of efficacy \\
\hline Tarenflurbil & Myriad Genetics/Lundbeck & $\gamma$-secretase modulator & Mild & Lack of efficacy
\end{tabular}

A $\beta$, amyloid- $\beta$; BACE, $\beta$-secretase; mAb, monoclonal antibody; pAb, polyclonal antibody. Adapted from Nat. Rev. Neurol. 15, 73-88; 2019, Springer Nature Limited; see article for more details, including summaries of results from failed phase II trials. 